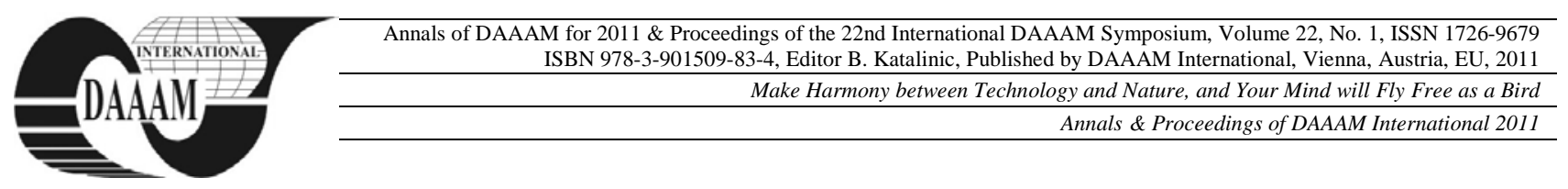

\title{
DESIGN OF SCAN MACHINE FOR IMAGE CATALOGING
}

\author{
ANDRS, O[ndrej] \& KOVAR, J[iri]
}

\begin{abstract}
This article deals with design of a scan machine for purpose of the image cataloging. The machine concenpt itself is based on well known Delta robot. The movable platform of scan machine is equipped by high-resolution camera. For automatic recognition of images on the paper is used particle swarm optimization technique. At the end of paper are shown experimental results of the laboratory machine testing.

Key words: scan machine, delta robot, PSO, soft-computing, parallel robot
\end{abstract}

\section{INTRODUCTION}

The image recognition by computer represents the current research problem and it is extensively investigated. A lot of studies have been made. The image screening with selection of a candidate set in the subimage that is similar to object class is used in (Arimura \& Hagita, 1996). The difference image entropy recognition system and the input image selection method are presented by (Jong-Bae Jeon et al., 2008). Other regognition methods are proposed by (Fei-Fei et al., 2004; Valach \& Kvas, 2010).

One of those ways is the particle swarm optimization (PSO) technique (Kennedy \& Eberhart, 1995). This method is widely used in all engineering applications, not only for computer vision tasks.

The image recognition described in this article uses the particle swarm optimization technique to find images on pages. This technique is inspired by the social behavior of swarm (Guochu Chen, 2010) and is applied in the control software of the scan machine. This machine is intended to cataloging of images in books. The main contribution of this study is to developed automatic device (the scan machine) for image recognition which should facilitate the cataloging.

The scan machine uses well known Delta robot conception. A mechanism with this conception is capable to realized translation with desired accuracy. The Delta robot was invented by Reymond Clavel at the EPFL (Ecole Polytechnique Fédérale de Lausanne, Switzerland). The purpose of this type of robot was to manipulate light and small objects at a very high speed (Clavel, 1990). The end effector of the robot is equipped with a camera, which is capable to zoom in and scans images.

\section{DESIGN OF MACHINE}

The design of intended machine was started by mechanical parts modeling in CAD software. The concept of the Delta robot uses parallelograms which allow the movable platform to remain at a fixed orientation with respect to the base. The movable platform is connected through three links to the fix base which is usually mounted above the workspace. The use of base mounted actuators and low mass links allows the movable platform to achieve high accelerations. This makes the Delta robot a perfect candidate for pick and place operations with high dynamics.
The suggested machine is prepared for different type of application. The movable platform is equipped with the high resolution Microsoft LifeCam NX-6000 camera which scans images on the required position. Due to design of the camera holder the camera lens axis has position offset to the platform center point which is compensated in a control system.

The design below brings the basic feasibility study for the laboratory model of the Delta robot which substitutes a real machine. The laboratory model (Fig. 1) constitutes miniature of the real scan machine and sustains full functionality. The constructed device represents mechatronic system (Hadas et al., 2010). The mentioned design solution for the development of mechatronic products is presented according to industrial guideline - VDI 2206.

\subsection{Control system}

The control system for this machine was developed in NI LabVIEW software environment. One of the big advantages of programming with this high-level tool is that we can use same algorithms for multiple platforms. Hence, the control system was implemented for evaluation board with the LM3S8962 microcontroller and it will also be used in the final application with NI CompactRIO control device.

The control system uses solution of the inverse kinematic task which is necessary for position control (Merlet, 2005).

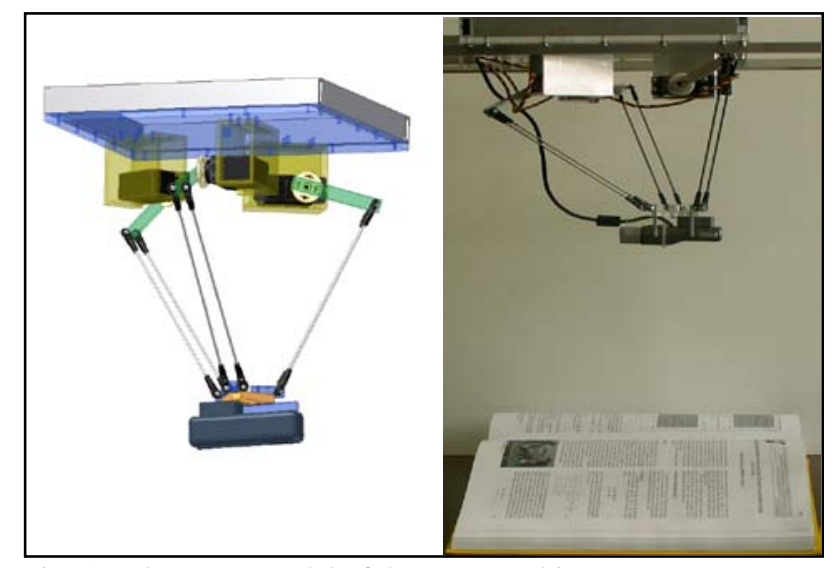

Fig. 1. Laboratory model of the scan machine

\section{PSO ALGORITHM}

The particle swarm optimization technique is well known soft-computing method based on the fact that the behavior of entire swarm is affected by the behavior of each individual (ie. fishes, birds etc.). If we want to describe movement of the particle in 2D space in mathematical terms, we will obtain:

$$
\begin{gathered}
\vec{V}_{i}(t+1)=w \vec{V}(t)+c_{1} r_{1}\left(\vec{X}\left(P_{i}\right)-\vec{X}_{i}(t)\right)+c_{2} r_{2}\left(\vec{X}\left(P_{G}\right)-\vec{X}_{i}(t)\right) \\
\vec{X}_{\mathrm{i}}(t+1)=\overrightarrow{\mathrm{X}}_{\mathrm{i}}(t)+\overrightarrow{\mathrm{V}}_{\mathrm{i}}(t+1)
\end{gathered}
$$


where $\mathrm{X}$ is a position and $\mathrm{V}$ is a velocity of the particle. Parameters c1, c2 and $\mathrm{w}$ describes the degree of individuality and depending on swarm, $\mathrm{r} 1$ and $\mathrm{r} 2$ are random numbers, $\mathrm{P}$ is the local (individual) maximum (in equation position of this maximum) of the particle, $P G$ is maximum of swarm and $i$ denotes the index of particles (individuals).

Particles are "moving" on a two-dimensional surface. This surface is the two-dimensional array which represents the preproccesed photo image of the page and contains numbers between 0 and 255. The optimization in this case means to find the image at this page. This ability of PSO depends on the evaluation function, which is:

$$
P=\sum_{i=-m}^{m} E\left(X_{X}+i ; X_{Y}\right)
$$

where $P$ denotes the evaluation of particle, $X_{X}$ is position of the particle in x-axis, $X_{Y}$ is position of the particle in y-axis and $E$ denotes the evaluation of pixel.

\subsection{Image preprocessing}

As we mentioned before, the page in software is represented by the two-dimensional array. But after the photographing of the page we obtain three 2D arrays (one for each color component - RGB). Therefore, for reasons of the calculation reduction the image is gray scaled.

\subsection{Boundary function}

The aim of this function is to find the boundary of the image at the photographed page. In this case is used the method based on searching for the contiguous area. That means we are searching for the end of image lines. Results are represented by border points of the image at the page.

\section{EXPERIMENTAL RESULTS}

The experiment was done with the laboratory version of scan machine with these parameters (Tab. 1.). The result of experiment is shown at Fig. 2. In the enlarged rectangle are shown output of PSO algorithm and the boundary function (dashed rectangle). The result was obtained after 1.7 seconds (AMD Phenom II X4 945 processor, 3GHz).

\begin{tabular}{|c|c|c|c|}
\hline $\mathbf{w}$ & 0.8 & $\mathbf{c}_{\mathbf{1}}$ & 0.35 \\
\hline $\mathbf{c}_{\mathbf{2}}$ & 0.75 & $\begin{array}{c}\text { Number of } \\
\text { iterations (T) }\end{array}$ & 50 \\
\hline $\begin{array}{c}\text { Number of } \\
\text { particles }\end{array}$ & 20 & $\begin{array}{c}\text { Resolution of } \\
\text { picture }\end{array}$ & $\begin{array}{c}1600 \times 1200 \\
\mathrm{px}\end{array}$ \\
\hline $\mathbf{m}$ & 20 & \multicolumn{2}{|c}{} \\
\cline { 1 - 3 } & &
\end{tabular}

Tab. 1. Table of PSO parameters

\section{CONCLUSIONS}

The application of scan machine for the image recognition was introduced in this paper. The scan machine is based on the Delta robot and uses the PSO algorithm for the image recognition. The control system of this machine was realized in NI LabVIEW. The feasibility of this attitude was experimentally tested and the result is shown at Fig. 2. The limitation of this approach is that the picture must be darker than the background. Further improvement will lie in extension of the movable platform by additional rotary axis for image deflection compensation and manufacturing of the real machine. The next step of improvement will be speed optimization of soft-computing method.

\section{ACKNOWLEDGEMENT}

This work is supported from research plan FSI-J-11-39, FSI-J-11-40/1373 and FSI-S-11-23.

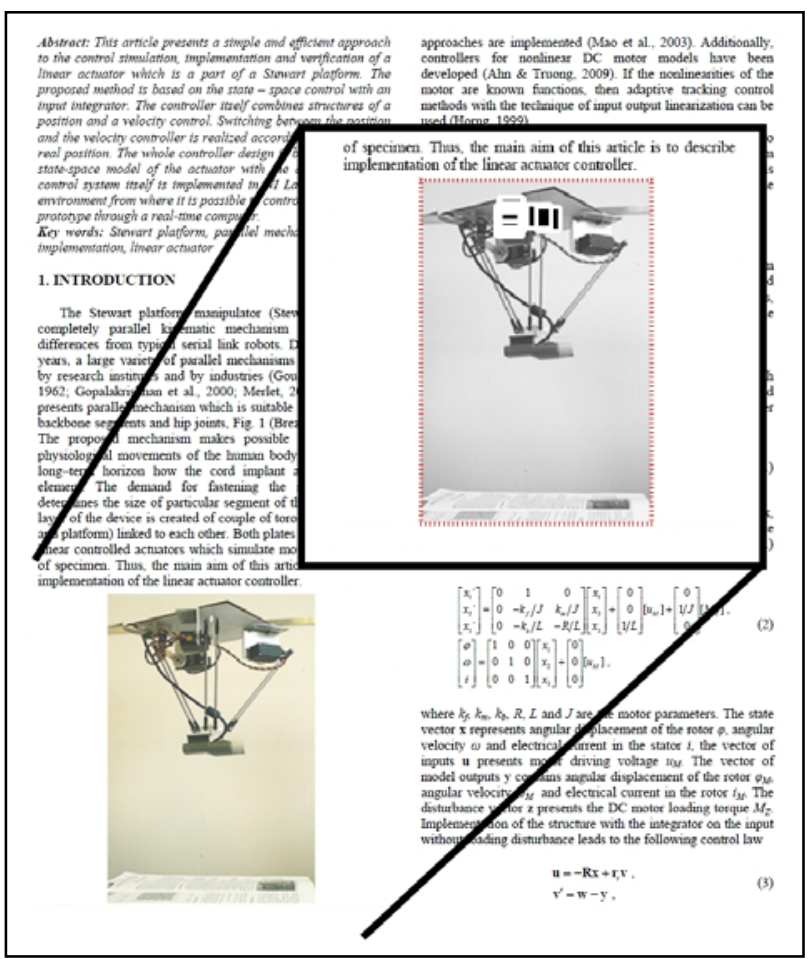

Fig. 2. The photographed image and in dashed rectangle are the output from PSO and the boundary function (white rectangles are particles)

\section{REFERENCES}

Arimura, K. \& Hagita, N. (1996). Feature space design for image recognition with image screening, Proceedings of the 13th International Conference on Pattern Recognition, 1996, pp.261-265 vol.2, ISBN: 0-8186-7282-X

Clavel, R. (1990). Device for the Movement and Positioning of an Element in Space, US Patent No. 4,976,582

Fei-Fei, L.; Fergus, R. \& Perona P. (2004). Learning generative visual models from few training examples: an incremental Bayesian approach tested on 101 object categories, Proc. of CVPR Workshop on Generative-Model Based Vision, 2004, ISSN: 1077-3142

Guochu Chen (2010). Simplified particle swarm optimization algorithm based on particles classification, Sixth International Conference on Natural Computation (ICNC), vol.5, pp.2701-2705

Hadas, Z.; Vechet, S.; Singule, V. \& Ondrusek, C. (2010) Development of Energy Harvesting Sources for Remote Applications as Mechatronic systems, Proceedings of EPEPEMC 2010 - 14th International Power Electronics and Motion Control Conference, pp.13-19, Ohrid, Macedonia

Jong-Bae Jeon; Jung-Hyun Kim; Jun-Ho Yoon \& Kwang-Seok Hong, (2088). Performance Evaluation of Teeth Image Recognition System Based on Difference Image Entropy, ICCIT '08 Third International Conference on Convergence and Hybrid Information Technology, vol.2, no., pp.967972, 11-13 Nov. 2008, ISBN: 978-0-7695-3407-7

Kennedy, J. \& Eberhart, R. (1995). Particle swarm optimization, Neural Networks, Proceedings., IEEE International Conference, vol.4, pp.1942-1948, 1995

Merlet, J. P. (2005). Parallel robots, 2nd Edition, Kluwer Academic Publishers, Dordrecht, ISBN 1402041322

Valach, S. \& Kvas, M. (2010). A Fast Camera with Online Image Evaluation, Annals of DAAAM for 2010 \& Proceedings of the 21st International DAAAM Symposium, 20-23rd October 2010, Zadar, Croatia, ISSN 1726-9679, ISBN 978-3-901509-73-5, Katalinic, B. (Ed.), pp. 06010602, Published by DAAAM International Vienna, Vienna 